\title{
Construction Modeling and Schedule Optimization for Deep Foundation Pit of Metro Station Based on Time-Space Effect and Risk Control
}

\author{
Guangming Yu, Xiankun Zeng, Yongjun Qin, Yongning Li, Yingnian Yu, and Xianguo Meng
}

\begin{abstract}
Metro stations are often built in the main urban area of the cities, its surrounding buildings are dense, the sources of risk are numerous. The construction of the metro station foundation pit is bound to have a certain impact on the adjacent buildings around. Therefore, it is necessary to scientifically follow the time-space effect of the construction of the foundation pit of the metro station, control the additional stress caused by the construction of the foundation pit of the metro station to the surrounding soil and buildings in the whole process and control the construction process of the foundation pit of the metro station scientifically. In order to ensure that the metro station foundation pit construction around the adjacent buildings do not cause harmful effects. Combined with a deep foundation pit engineering example of a metro station in a city, the change of stratum stress in the process of excavation and support of deep foundation pit are simulating by using the 3D finite element software MIDAS GTS NX (New eXperience of Geo-Technical analysis System). On this basis, the construction schedule of the deep foundation pit is optimized by using the Critical Chain Method, the scientific and advanced management mode of the deep foundation pit construction of the metro station based on the risk control and time-space effect is obtained, so as to evaluate the time-space effect and the control effect of the deep foundation pit construction. The integration of mechanical modeling, mechanical analysis, risk management and schedule optimization is realized.
\end{abstract}

Index Terms-Time-space effect, risk control, Deep foundation pit of metro station, construction modeling, schedule optimization.

\section{INTRODUCTION}

With the rapid development of urban scale and modernization, the ground traffic becomes more and more crowded, the original ground transportation facilities cannot

Manuscript received September 26, 2018; revised January 20, 2019. This work was supported in part by the National Natural Science Foundation of China under Grant 51674150 and the Construction of Science and Technology Projects in Urumqi under Grant 2016002.

Guangming Yu, Yongjun Qin and Yongning Li are with College of Architecture and Civil Engineering, Xinjiang University, Urumqi 830047, China (e-mail: 861692371@qq.com, 470993227@qq.com, 1179742980@qq.com).

Guangming Yu and Xiankun Zeng are with School of Civil Engineering, Cooperative Innovation Center of Engineering Construction and Safety in Shandong Blue Economic Zone, Qingdao University of Technology, Qingdao 266033, China (e-mail: yu-guangming@263.net, 2603696765@qq.com).

Yingnian Yu is with Qingdao Company, Zhongqi Jiaojian Group Co., Ltd., Qingdao 266300, China (e-mail: 1795556786@qq.com).

Xianguo Meng is with the Xinjiang Wujing Infrastructure Construction Management Co., Ltd., Urumqi 830000, China 38678, Germany (e-mail: 1657008794@qq.com). meet the needs of urban development. The metros can effectively alleviate this kind of urban ground traffic pressure. However, on the one hand, the metro is often built in the main urban area of the city, especially in the bustling area of the city, its surrounding buildings and pipelines and other risk sources are larger. On the other hand, the foundation pit engineering of metro station is also developing in the direction of larger area and deeper depth, the geological conditions and hydrological conditions are more complex. Therefore, the construction of deep foundation pit of metro station is a complex system engineering. If the construction process is not properly managed, it will cause damage to the surrounding environment such as buildings and underground pipelines, it will not only bring about huge economic losses. It will also have a negative social impact. Therefore, in the construction of deep foundation pit of metro station, the construction process of foundation pit of metro station should be controlled scientifically, the time-space effect of the construction of the deep foundation pit of the metro station should be followed scientifically. In order to ensure that the construction of foundation pit of metro station around the adjacent buildings do not have harmful effects.

In the application of time-space effect theory of deep foundation pit construction, Peck and Terzaghi studied the displacement mechanism of surrounding soil caused by foundation pit excavation [1], [2]. James analyzed the deformation control of foundation pit to reduce the damage to surrounding environment, putting forward the deformation control theory of foundation pit for the first time [3]. Mana and Clough researched the influence of excavation size on foundation pit deformation by using the finite element method [4]. F. H. Lee et al. combined finite element method and field monitoring data analysis to verify the corner effect of foundation pit deformation [5]. J. L. Yu et al. considered the deformation and internal force characteristics of retaining structure under the time-space effect of foundation pit construction [6]. J. JIA solved a large number of deep foundation pits in soft soil area by using the method of time-space effect of foundation pit [7]. Z. M. WU et al. analyzed the interaction between soil nails and soil by using the space-time effect principle [8]. G. P. WANG et al. studied the space-time effect law of soil around foundation pit in soft soil area and the calculation method of internal force and deformation of supporting structure based on the finite element theory of bar system [9]. G. N. Zhou analyzed the monitoring data of railway station construction in a city [10]. Y. J. LI et al. studied the regularity of surrounding ground settlement caused by unloading soil of foundation pit 
excavation, putting forward the calculation model of foundation pit settlement [11]. At the same time, in the process of risk management of foundation pit construction, there are some problems such as inaccurate prediction of construction period, Parkinson's law and student syndrome in project management. For this reason, Israeli physicist Goldratt proposed the Critical Chain Method based on constraint theory which is currently widely used in schedule control of project construction [12]. According to the idea of spatial mapping of interval grey numbers, the whitening weight function is introduced to represent the distribution information of interval grey numbers [13]. Y. W. Zhang et al. combined the management method of PDCA Circle with tunnel construction schedule control and put forward the corresponding rolling flexible control method of tunnel construction schedule [14]. Z. Zhang et al. adopted risk analysis method and put forward the Critical Chain Technology [15].

As can be seen from the above research, there is a lack of research on the combination of time-space effect theory and risk control theory with the deep foundation pit of metro stations. Now, the construction technology and risk control requirements of the deep foundation pit of metro station are more stringent and accurate. Based on the simulation of the ground stress change during the excavation and support of deep foundation pit by using the 3D finite element software MIDAS GTS NX, the construction schedule of the deep foundation pit is optimized by using the Critical Chain Method, the scientific and advanced management model of the deep foundation pit construction of metro station based on risk control and space-time effect is constructed. The time-space effect and control effect of the construction of the deep foundation pit are expected to reduce the construction risk and cost and improve the quality of the project.

\section{A. Related Basic Theories}

\section{1) Time-space effect theory of foundation pit engineering}

The so-called time-space effect of foundation pit engineering refers to the quantitative consideration of the influence of construction factors on the deformation of foundation pit and surrounding soil. It has the following two main characteristics.

Firstly, it is the time effect of foundation pit construction. According to the previous summary, the excavation method of layering, block, symmetry, balance and time-limited is usually adopted in foundation pit excavation, which can effectively mobilize the spatial effect of stratum, thus has the advantages of reducing stress level, controlling deformation and displacement, reducing the excavation of each construction condition until the end of bracing. Time, as far as possible to reduce the exposure time of foundation pit without support, can significantly reduce the deformation of the retaining structure, especially when the exposure time of no support is less than 24 hours.

Secondly, it is the space effect of foundation pit construction, the shape of plane section of foundation pit excavation has irregular characteristics, different plane section shape of foundation pit has different deformation laws, in the same foundation pit, the construction of the foundation pit at different parts of the deformation law is different, such as the deformation of the long side of the foundation pit is larger than the deformation of the short side. There are also different deformation characteristics of the foundation pit Therefore, in order to achieve the purpose of construction safety, the theory of time-space effect has been widely used in the control of deformation of foundation pit engineering.

In the field of geotechnical engineering at this stage, numerical simulation method has always been one of the most effective means to study the space-time effect of deep foundation pit. Among them, the finite element analysis software Midas GTS NX is suitable for deep foundation pit construction stage. It has many advantages such as diversified modeling methods, the latest solver, high-speed analysis and processing speed, having been widely recognized by scholars.

\section{B. Risk Control Theory of Foundation Pit Engineering}

\section{2) Whitening weight function}

The key of the project schedule is to estimate the duration of the project process activities. Some projects have abundant data preparation and known project duration. In this case, the project schedule can be formulated directly by CCM method, some projects are relatively single, the relevant historical data are relatively small, the information is not available. Completely, in this case, subjective estimates are usually made based on managers or developers' own rich management experience and level of risk prediction. In view of this situation, the trapezoidal whitening weight function in the grey model algorithm is introduced, the time estimation of project process activity is improved by combining the trapezoidal whitening weight function. The characteristics of the grey model are as follows: the forecasting accuracy is high, the sample is less, the sample does not need to have regular distribution, the calculation amount of the grey model is relatively small, so it is suitable for forecasting and evaluation. According to the characteristics of grey model, this method can be used to estimate the duration of project activity which is lack of historical experience. The time interval of ladder whitening weight function is used to estimate the duration of project activity. The function graph is shown in Fig. 1.

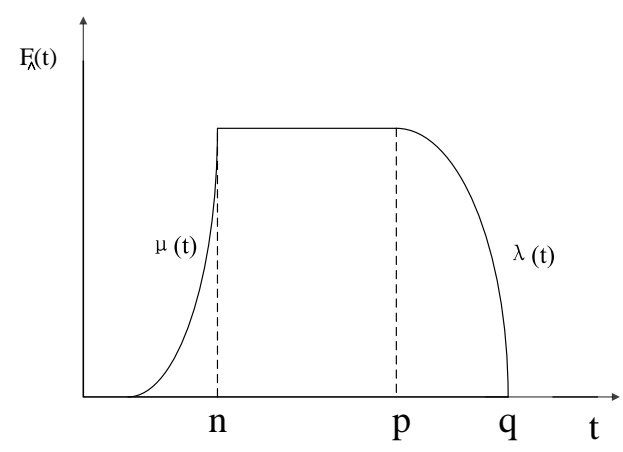

Fig. 1. Trapezoidal whitening weight function curve.

The horizontal axis $t$ represents the duration of the project process, the longitudinal axis $F_{\Lambda}(t)$ indicates the possibility of t. The function $F_{\Lambda}(t)$ is divided into three sections, $\mu(t)$ is exponential decreasing function, $\lambda(t)$ is decreasing function, the interval $[\mathrm{n}, \mathrm{p}]$ represents the most probable 
interval of the duration of the project activity. m represents the most optimistic estimate of the project process duration, $n$ represents the minimum of the most likely completion time of the project process duration, $p$ represents the maximum of the most likely completion time of the project process duration, $q$ represents the most pessimistic estimate of the project process duration. As shown in Fig. 1, the nonlinear functions $F_{\wedge}(t)$ are in the intervals $[m, n]$ and $[p, q]$.

$$
F_{\Lambda}(t)=\left\{\begin{array}{l}
0, t<m \\
\mu(t), m \leq t<n \\
1, n \leq t \leq p \\
\lambda(t), p<t \leq q \\
0, t>q
\end{array}\right.
$$

Although theoretically it can be used $F_{\wedge}(t)$ to estimate the process duration of a project, because of the nature of the nonlinear function, it is very complicated to calculate the process duration. For convenience and calculation, the nonlinear function $F_{\wedge}(t)$ is transformed. The linear function $F \wedge(t)$ is shown as follows.

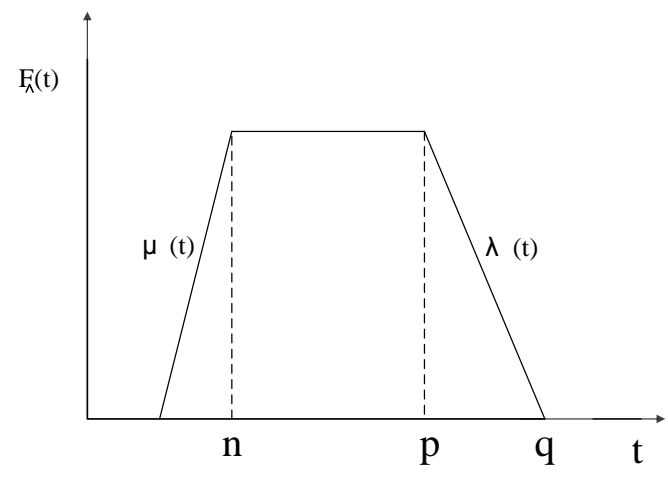

Fig. 2. Linear function curve.

By mathematical calculation, the function $F_{\Lambda}(t)^{\prime}$ after deformation is

$$
F_{\Lambda}(t)^{\prime}=\left\{\begin{array}{l}
\frac{t-m}{n-m}, m \leq t<n \\
1, n \leq t \geq p \\
\frac{q-t}{q-p}, p<t \leq q \\
0, \text { else }
\end{array}\right.
$$

The expected duration $E(X)$ of process duration $X \in[1, n]$ can be calculated by probability calculation.

$$
E(X)=\frac{\int_{m}^{q} F(t)^{\prime} t d t}{\int_{m}^{q} F(t)^{\prime} d t}=\frac{2 m_{i}+n_{i}+\mathrm{p}_{i}+q_{i}}{6}
$$

The whitening weight function is used to estimate the process duration, the initial estimated duration (D) is calculated. The other schedule parameters are calculated with the following Critical Chain Method.

\section{Critical Chain Method}

Critical Chain Theory holds that the completion time of a project depends on the length of the Critical Chain and the management of the Critical Chain in the project. It is a new project management idea based on the critical path, which solves the schedule management problems of those resource-constrained projects and controls the project progress by controlling the Critical Chain. The goal of project schedule management of Critical Chain is to ensure that the project can be completed in the shortest time with limited resources. This goal means that the bottleneck in the implementation process of the project is the longest link in each path of the project, that is, the Critical Chain.

The traditional Critical Chain Method regards half of the planned time as the safety time and half of the safety time as the Project Buffer (PB). The improved method takes into account the risk time of project completion, not using a single absolute estimate of the time limit, but reducing the error caused by the estimated value of the time limit through the probability coefficient of the process.

Critical Chain Technology considers the resource constraints and the uncertainties of project duration, introducing the buffer concepts and buffer management Buffers are inserted into any project schedule to weaken the safety time of each process and avoid resource conflicts and uncertainties. The Critical Chain is the critical path after resolving resource conflicts and inserting buffers. Critical Chain Technology avoids project schedule delay caused by Critical Chain process problems by inserting the $\mathrm{PB}$ at the end of Critical Chain, inserting Feeding Buffer (FB) before non-Critical Chain imports Critical Chain process to avoid the impact of non-Critical Chain process delay on Critical Chain process. The buffer size of Critical Chain Technology is determined according to the duration of the corresponding the process activities of Critical Chain.

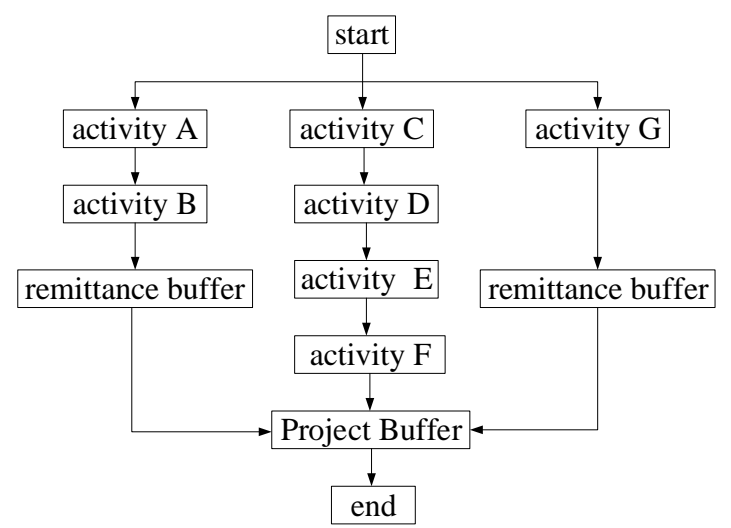

Fig. 3. Critical Chain process

Because the traditional project schedule management usually adds safety time to estimate the time limit of active tasks in the project, so the time limit estimation is a very conservative and stable method, but this often causes a waste of time cost, which is very unreasonable. The new solution is as follows: traditional method is used to calculate the initial estimate value of the project process duration. The whitening weight function of grey model is used to calculate the time limit, the improved time estimate $d$ is calculated. The safety time $\Delta=D-d$ of each process is calculated. Let the initial 
estimate value of each process duration in the Critical Chain be $D_{i}^{M}$, the most satisfactory estimate value of the process duration is $d_{i}^{M}$, the process safety time is $\Delta_{i}^{M}=D_{i}^{M}-d_{i}^{M}$, the initial estimate value of each process duration in the non-Critical Chain is $D_{j}^{N}$, the most satisfactory estimate value of the process duration is $d_{j}^{N}$, the process safety time is $\Delta_{j}^{N}=D_{j}^{N}-d_{j}^{N}$, where $\mathrm{M}$ represents the Critical Chain in the project. $\mathrm{N}$ represents the collection of processes on a non-Critical Chain in a project.

The buffer coefficient $\alpha$ is calculated as follow.

$$
\alpha=\frac{D-d}{D}
$$

Among them, the probability coefficient of delay may be expressed. The buffer coefficient $\alpha_{i}^{M}$ of each process activity on the Critical Chain is

$$
\alpha_{i}^{M}=\frac{D_{i}^{M}-d_{i}^{M}}{D_{i}^{M}}
$$

The buffer coefficient $\alpha_{j}^{N}$ of each process activity on a non-Critical Chain is

$$
\alpha_{j}^{N}=\frac{D_{j}^{N}-d_{j}^{N}}{D_{j}^{N}}
$$

Therefore, the formula (5) and the safety time of Critical Chain are multiplied to get the PB formula, which is as follow.

$$
\begin{aligned}
& P B=\sum_{i \in M} \alpha_{i}^{M} \times\left(D_{i}^{M}-d_{i}^{M}\right)=\sum_{i \in M} \frac{\left(D_{i}^{M}-d_{i}^{M}\right)^{2}}{D_{i}^{M}} \\
& =\sum_{i \in M} \frac{\left(\Delta_{i}^{M}\right)^{2}}{D_{i}^{M}}
\end{aligned}
$$

By multiplying formula (6) with non-Critical Chain safety time, the formula of FB is obtained as follow.

$$
\begin{aligned}
& \left.F B=\sum_{j \in N} \alpha_{j}^{N} \times D_{j}^{N}-d_{j}^{N}\right)=\sum_{j \in N} \frac{\left(D_{j}^{N}-d_{j}^{N}\right)^{2}}{D_{j}^{N}} \\
& =\sum_{j \in N} \frac{\left(\Delta_{j}^{N}\right)^{2}}{D_{j}^{N}}
\end{aligned}
$$

The buffer size is calculating by the mathematical probability knowledge. The algorithm is concise. In the calculation process, the traditional method and the improved method are used to estimate the time limit respectively. The risk time of the project completion is considered, the single absolute time limit is not used. Through the probability coefficient of the process to reduce the error caused by the estimated duration, so that the estimated duration closer to the actual value of the process, more accurate.
In addition, when the safety time $\Delta$ equals the estimated time $d$, i.e. $D-d=d$, that is, when the improved time estimate is one half of the traditional time estimate, the buffer coefficient of the process is provided, $\alpha=\frac{D-d}{D}=\frac{\frac{1}{2} D}{D}=\frac{1}{2}$, where the $P B=\frac{1}{2} \sum_{i \in M} \Delta t_{i}^{M}$, the FB is $F B=\frac{1}{2} \sum_{j \in N} \Delta t_{j}^{N}$, that is, when $\mathrm{D}$ is one half of $\mathrm{D}$, the buffer coefficient $\alpha$ is 0.5 . The method becomes a special form of shear paste method.

\section{COMPREHENSIVE APPLICATION OF TIME-EFFECT THEORY AND RISK CONTROL THEORY IN DEEP FOUNDATION PIT CONSTRUCTION}

\section{A. Project Overview}

The Erdaoqiao Station of Urumqi Metro Line No.1 is located under Jiefang South Road in Tianshan District of Urumqi City, it is arranged in a north-south direction. In the southwest quadrant of the station, there are the Unity Theatre, the National Night Market and the Commercial and Residential Buildings. In the Northwest quadrant, there are Nanda Temple, Xiong Ying International Hotel and Erdaoqiao Exchange Market. In the Northeast quadrant, there are No. 4 Building of Xinjiang International Baza and No. 1 Building of Xinjiang International Baza in the Southeast quadrant. YDK2+463.382 to YDK2+669.382 is the design starting point mileage of the station. The scale of the station is an underground two-storey island platform station, the construction is carried out by open-cut method. The foundation pit is constructed by open-cut method, the retaining structure is composed of retaining piles and steel pipe internal support system. The length of the foundation pit is $206 \mathrm{~m}$, the width of the standard section is $21.6 \mathrm{~m}$, the depth of the floor is about $19.53 \mathrm{~m}$. The total excavation volume of the foundation pit excavation at the Erdaoqiao Station is about $9.5 \mathrm{~m}$, the average daily muck handling is $710 \mathrm{~m}$.

\section{B. 3D Numerical Simulation of Deep Foundation Pit}

Before establishing the model, we must first select the model type as $3 \mathrm{D}$, the gravity direction as $\mathrm{Z}$, the system unit in the toolbar, the internal force unit as $\mathrm{kN}$, the displacement unit as $\mathrm{m}$, the other parameters as well as the initial parameters are defaulted, as shown in Fig. 4.

According to a large number of numerical simulation results in the past and practical engineering experience in the field, the influence range of deep foundation pit excavation in the vertical direction is 1 2 times of the excavation depth of the foundation pit, the influence range in the horizontal direction is 3 5 times of the excavation depth. Therefore, the final model size is $375 \mathrm{~m}$ in length, $165 \mathrm{~m}$ in width and $60 \mathrm{~m}$ in depth.

Before establishing the model, draw the foundation pit model plan in CAD, as shown in Fig. 5, then import the 2D DXF plan into the simulation software, or draw the plan directly in the simulation software, expand the $3 \mathrm{D}$ entity with the model plan which is imported or generated, then control the size of the foundation pit entity by the linear gradient method to make the network. The length of the cell is divided 
into 2-4 $\mathrm{m}$, followed by automatic meshing operation. The length of the cell increases from the foundation pit to the surrounding radiation. This method can reduce the number of elements as much as possible under the premise of guaranteeing the precision of simulation calculation, so as to improve the calculation efficiency and reduce the calculation time. The model is divided into 232890 units. The model divides the grid as shown in Fig. 6.

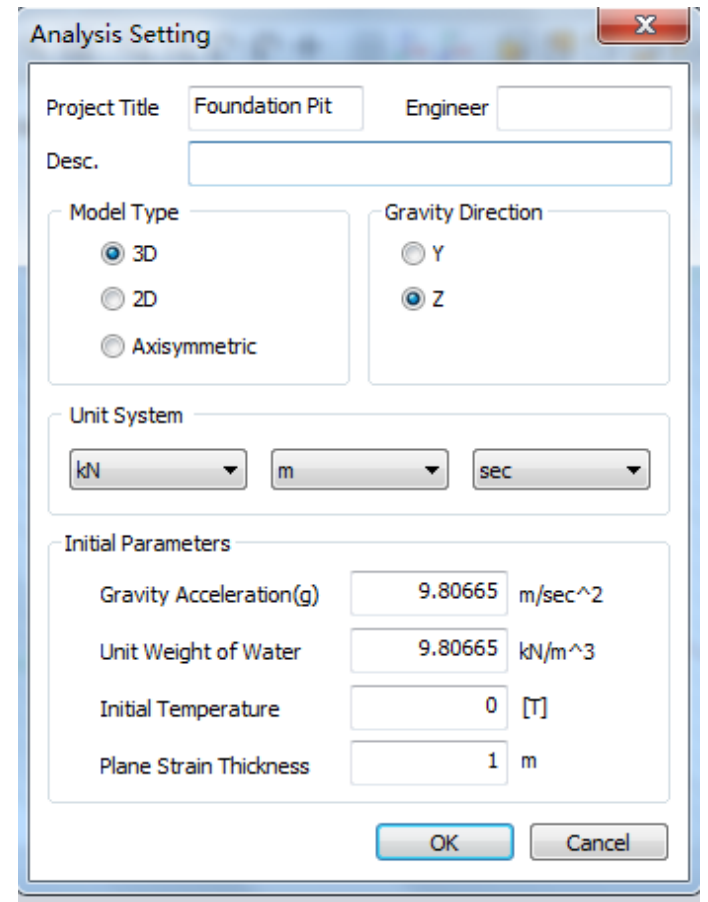

Fig. 4. Analysis of setup parameter interface.

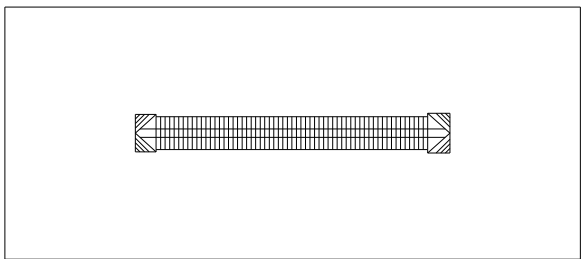

Fig. 5. 2D plane Fig. of deep foundation pit

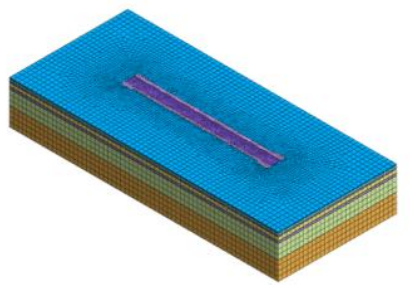

Fig. 6. 3D mesh of foundation pit model.

According to the actual engineering conditions, the directional bracing in the direction $\mathrm{X}$ is set on the left and right sides of the foundation pit model, the directional bracing in the direction $\mathrm{Y}$ is set on the front and back sides of the foundation pit model, the directional bracing in the direction $\mathrm{Z}$ is set on the bottom surface of the model, the displacement in the top three directions of the model does not impose any direction of determination. To support. After the model boundary is set, the dead load is defined, the dead load coefficient is set to -1 . The displacement boundary condition is set as shown in Fig. 7.
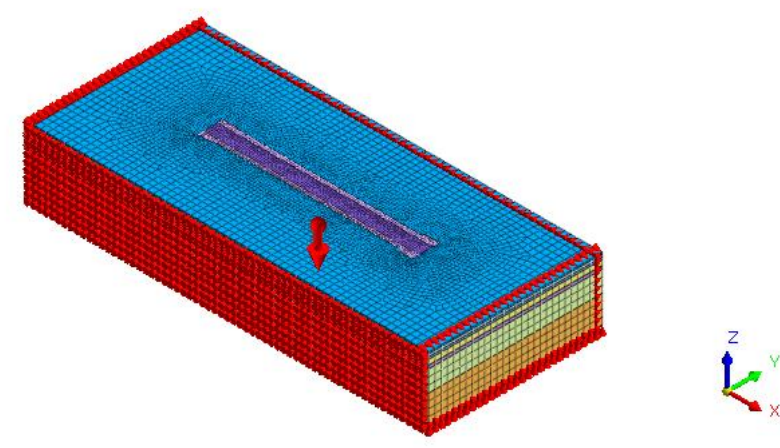

Fig. 7. Boundary condition of model displacement.

Foundation pit excavation is strictly in accordance with the time-space effect theory, zoning, layering, sectional construction, strictly in accordance with the "first support and then excavation, prohibit over-excavation" principle for safe construction. The rock and soil inside the foundation pit are excavated vertically in four layers; the excavation is carried out horizontally in the sequence of excavating the soil on both sides of the slot after excavating the part of the intermediate slot at the same time; if the excavation is carried out vertically in accordance with the actual project, the excavation is divided into seven throttling water sections, each additional throttling water section, the construction Construction stage will be doubled, resulting in excessive calculation. The result is too long and so on. Therefore, the excavation of multiple sections of the same layer is regarded as one step excavation and forming at one time. When excavating the soil, in order to erect the retaining structure safely in the pit, the earthwork should be excavated to $0.5 \mathrm{~m}$ below the bottom of the steel pipe support, the exposure time of the unsupported area of the foundation pit should be reduced as far as possible. The specific size of the excavation area is shown in Fig. 8, the longitudinal delamination depth is shown in Fig. 9.

Concrete simulation of construction stags as follows:

Initial stress stage: activate all rock and soil, automatic displacement constraint and self-weight, check the displacement to zero.

Construction stage 1: activate the equivalent stiffness of the diaphragm wall and excavate the first layer of soil in the middle groove area.

Construction stage 2: when excavating the two sides of the first layer of soil in the trough area at the same time, it is necessary to excavate to $0.5 \mathrm{~m}$ below the bottom of the first support.

Construction stage 3: erecting the first purlin (crown beam) and the first transverse support, activating and prestressing it;

Construction stage 4: excavating second layers of soil in the middle groove area;

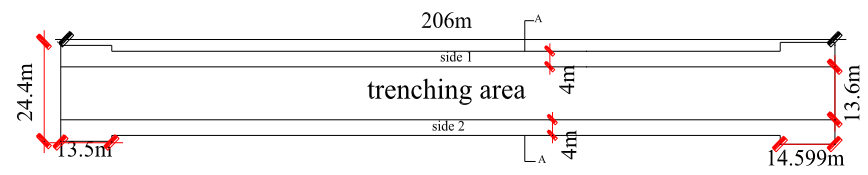

Fig. 8. Horizontal excavation plan for foundation pit. 
Construction stage 5: when excavating both sides of the second layer of soil in the bracing area, the soil must be excavated to $0.5 \mathrm{~cm}$ below the bottom of the second support.

Construction stage 6: the second purlin (i. e. the first waist beam) and the second cross support are erected and activated to apply prestress.

Construction stage 7: excavating third layers of soil in the middle groove area.

Construction stage 8: when excavating both sides of the 3 rd layer of soil in the bracing area simultaneously, the soil must be excavated to $0.5 \mathrm{~cm}$ below the bottom of the third support.

Construction stage 9: the 3rd purlin (i. e. the second waist beam) and the 3rd transverse support are erected, activated and prestressed.

Construction stage 10: excavating 4th layers of soil in the middle groove area.

Construction stage 11: excavation of 4 th sides of soil at the same time.

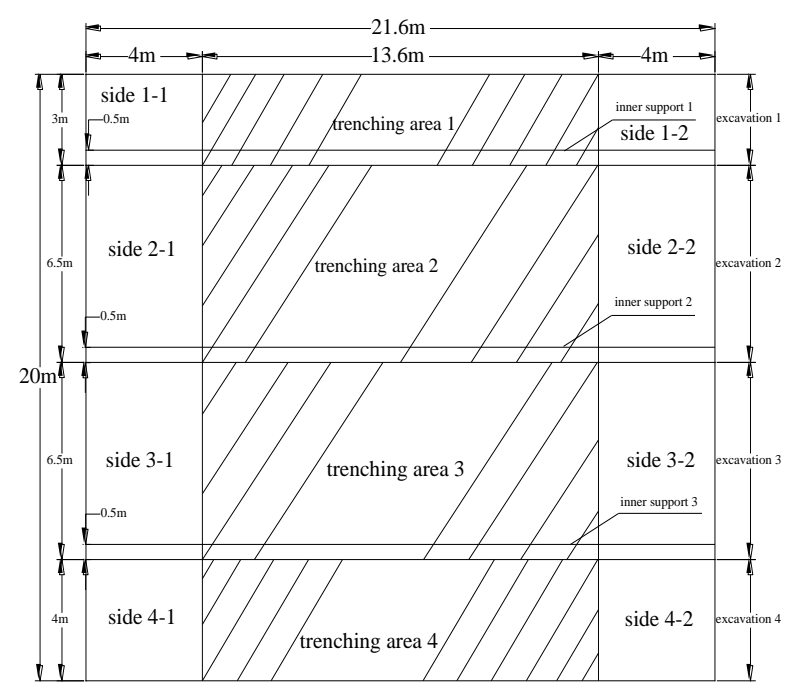

Fig. 9. Vertical section of A-A section of foundation pit.

TABLE I: OPTIMIZATION PLAN FOR CONSTRUCTION SCHEDULE OF DEEP FOUNDATION PIT

\begin{tabular}{|c|c|c|c|c|c|c|c|c|}
\hline operation sequence & operation name & $\mathrm{m}$ & {$[\mathrm{n}, \mathrm{p}]$} & q & $\mathrm{D}$ & $\mathrm{d}$ & $v$ & $\alpha$ \\
\hline 1 & slot the 1st layer & 8 & {$[10,14]$} & 16 & 11 & 9 & 2 & 0.19 \\
\hline 2 & 1 tier 2 st section & 4 & {$[6,10]$} & 12 & 8 & 6 & 2 & 0.25 \\
\hline 3 & 1 tier 2 nd section & 2 & {$[4,8]$} & 10 & 6 & 4 & 2 & 0.33 \\
\hline 4 & 1 tier 3 rd section & 1 & {$[3,7]$} & 9 & 5 & 3.5 & 1.5 & 0.30 \\
\hline 5 & 1 tier 4 th section & 2 & {$[4,8]$} & 10 & 6 & 4 & 2 & 0.33 \\
\hline 6 & 1 tier 5 th section & 2 & {$[4,8]$} & 10 & 6 & 4 & 2 & 0.33 \\
\hline 7 & 1 tier 6 th section & 2 & {$[4,8]$} & 10 & 6 & 4 & 2 & 0.33 \\
\hline 8 & 1 tier 7 th section & 4 & {$[6,10]$} & 12 & 8 & 6 & 2 & 0.25 \\
\hline 9 & slot the 2 nd layer & 8 & {$[10,14]$} & 16 & 11 & 9 & 2 & 0.19 \\
\hline 10 & 2 tier 1 st section & 3 & {$[5,9]$} & 11 & 7 & 5 & 2 & 0.28 \\
\hline 11 & 2 tier 2 nd section & 2 & {$[4,8]$} & 10 & 6 & 4 & 2 & 0.33 \\
\hline 12 & 2 tier 3 rd section & 1 & {$[3,7]$} & 9 & 5 & 3.5 & 1.5 & 0.30 \\
\hline 13 & 2 tier 4 th section & 2 & {$[4,8]$} & 10 & 6 & 4 & 2 & 0.33 \\
\hline 14 & 2 tier 5 th section & 2 & {$[4,8]$} & 10 & 6 & 4 & 2 & 0.33 \\
\hline 15 & 2 tier 6 th section & 2 & {$[4,8]$} & 10 & 6 & 4 & 2 & 0.33 \\
\hline 16 & 2 tier 7 th section & 4 & {$[6,10]$} & 12 & 8 & 6 & 2 & 0.25 \\
\hline 17 & slot the 3rd layer & 8 & {$[10,14]$} & 16 & 11 & 9 & 2 & 0.19 \\
\hline 18 & 3 tier 1 st section & 3 & {$[5,9]$} & 11 & 7 & 5 & 2 & 0.28 \\
\hline 19 & 3 tier 2 nd section & 2 & {$[4,8]$} & 10 & 6 & 4 & 2 & 0.33 \\
\hline 20 & 3 tier 3 rd section & 1 & {$[3,7]$} & 9 & 5 & 4 & 1.5 & 0.30 \\
\hline 21 & 3 tier 4 th section & 2 & {$[4,8]$} & 10 & 6 & 4 & 2 & 0.33 \\
\hline 22 & 3 tier 5 th section & 2 & {$[4,8]$} & 10 & 6 & 4 & 2 & 0.33 \\
\hline 23 & 3 tier 6 th section & 2 & {$[4,8]$} & 10 & 6 & 4 & 2 & 0.33 \\
\hline 24 & 3 tier 7 th section & 4 & {$[6,10]$} & 12 & 8 & 6 & 2 & 0.25 \\
\hline 25 & slot the 4 th layer & 10 & {$[12,16]$} & 18 & 13 & 11 & 2 & 0.15 \\
\hline 26 & 4 tier 1 st section & 4 & {$[6,10]$} & 12 & 8 & 6 & 2 & 0.25 \\
\hline 27 & 4 tier 2 nd section & 2 & {$[4,8]$} & 10 & 6 & 4 & 2 & 0.33 \\
\hline 28 & 4 tier 3rd section & 1 & {$[3,7]$} & 9 & 5 & 4 & 1.5 & 0.30 \\
\hline 29 & 4 tier 4 th section & 2 & {$[4,8]$} & 10 & 6 & 4 & 2 & 0.33 \\
\hline 30 & 4 tier 5 th section & 2 & {$[4,8]$} & 10 & 6 & 4 & 2 & 0.33 \\
\hline 31 & 4 tier 6 th section & 2 & {$[4,8]$} & 10 & 6 & 4 & 2 & 0.33 \\
\hline 32 & 4 tier 7 th section & 4 & {$[6,10]$} & 12 & 8 & 6 & 2 & 0.25 \\
\hline
\end{tabular}

The vertical displacement cloud images of four construction stages at 2, 5, 8 and 11 are extracted, as shown in Fig. 10. The vertical displacement of each area inside and outside the deep foundation pit can be understood intuitively and scientifically from the displacement nephogram. As Erdaoqiao metro station is a narrow and deep structure, the construction has a great influence on the long side and the short side of the foundation pit, more attention should be paid to the long side of the foundation pit. At the same time, the numerical simulation results are compared with the measured data to verify the practicability of the numerical simulation. On this basis, the construction schedule can be quantitatively and effectively controlled in time and space, the

corresponding protective measures are taken for the key and difficult points in the construction process, so that the whole foundation pit is in a safe state, so as to achieve the goal. The organic unity of social benefits and economic benefits.

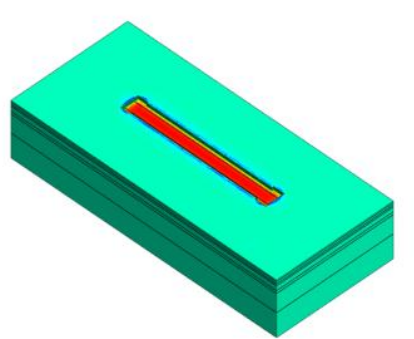

(a) construction stage 2

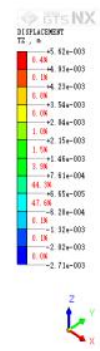

k 


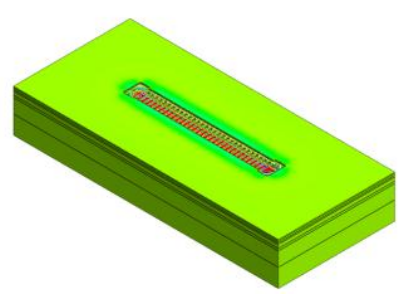

(b) Construction stage 5

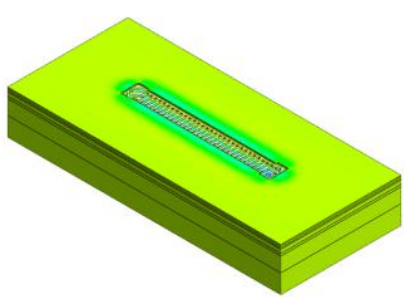

(c) construction stage 8

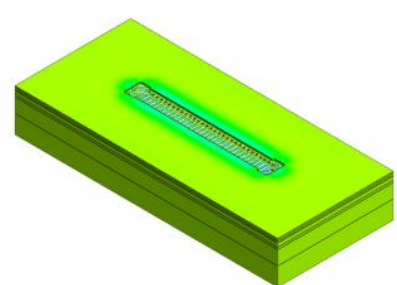

(d) construction stage 11

Fig. 10 Vertical displacement cloud map.

\section{Construction Progress Optimization plan}

Based on the above 3D numerical analysis, the optimization Critical Chain method is used to optimize the schedule of deep foundation pit project. The foundation pit activity can be divided into 32 activities. From the gray fuzzy whitening weight function, 32 specific initial construction time D can be obtained, as shown in the table below.

The PB from the above table is

$$
\begin{aligned}
& P B=\sum_{i \in M} \alpha_{i}^{M} \times\left(D_{i}^{M}-d_{i}^{M}\right)=\sum_{i \in M} \frac{\left(D_{i}^{M}-d_{i}^{M}\right)^{2}}{D_{i}^{M}} \\
& =\sum_{i \in M} \frac{\left(\Delta_{i}^{M}\right)^{2}}{D_{i}^{M}}=13(\text { days })
\end{aligned}
$$

The FB of the project is

$$
\begin{aligned}
& F B=\sum_{j \in N} \alpha_{j}^{N} \times\left(D_{j}^{N}-d_{j}^{N}\right)=\sum_{j \in N} \frac{\left(D_{j}^{N}-d_{j}^{N}\right)^{2}}{D_{j}^{N}} \\
& =\sum_{j \in N} \frac{\left(\Delta_{j}^{N}\right)^{2}}{D_{j}^{N}}=4(\text { days })
\end{aligned}
$$

By analyzing, the $P B$ is 13 days, indicating that if there is a problem in the process on the Critical Chain, it will lead to project schedule delay, the time available for schedule delay will not affect the progress of the Critical Chain has 13 days, the $F B$ is 4 days, if there is a delay in the process on the non-Critical Chain, to avoid the impact of time on the Critical Chain, there are 4 days.

\section{CONCLUSION}

By using numerical simulation method, the internal and external stability of deep foundation pit can be understood intuitively and scientifically in time and space, the practicability of numerical simulation can be verified by comparing the numerical simulation results with the measured data. On this basis, the construction schedule can be controlled quantitatively and effectively in time and space. Corresponding protective measures are taken for the key and difficult points in the construction process, so that the whole foundation pit is in a safe state, so as to realize the organic unity of social and economic benefits.

Based on the Critical Chain and combined with the grey fuzzy whitening weight function, the project scheduling method is more in line with the actual project management than the traditional project scheduling method. It overcomes the Parkinson's Law, Student Syndrome and Punishing Delay Only in project management. And the disadvantages of those who have quality problems.

The analysis of an example shows that the model and the method are effective, it can also be flexibly implemented in the case of resource change and schedule deviation. This research method is helpful to improve the schedule optimization and adjustment control level of large-scale construction projects and has a good application prospect.

\section{ACKNOWLEDGMENT}

This work was financially supported by the National Natural Science Foundation of China (51674150) and the Construction of Science and Technology Projects in Urumqi (2016002).

\section{REFERENCES}

[1] R. B. Peck, "Earth pressure measurements in open cut engineering," ASCE, 1943, Vol.108.

[2] R. B. Peck, "Deep excavations and tunneling in soft ground," Proceedings of 7th International Conference 011 Soil Mechanics and Foundation Engineering. State of-Art Volume Mexico City, 1969, pp: 225-290.

[3] Yao J T P, “Concept of structural control," Journal of the Structural Division, 1972, vol. 98, no. 7, pp. 1567-1574.

[4] A. I. Mana, G. W. Clough, "Prediction of movements for braced cuts in clay," Journal of Geotechnical Engineering, ASCE, 1981, vol. 107, no. 6, pp. 759-777.

[5] F. H. Lee, K. Y. Yong, and K. C. N. Quan, "Effects of corners in strutted excavations: field monitoring and case histories," Journal of Geotechnical and Geoenvironmental Engineering, ASCE, 1998, vol. 124, no. 4, pp. 339-349.

[6] J. L. Yu and X. N. Gong, "Spatial behavior analysis of deep excavation," Chinese Journal of Geotechnical Engineering, 1999, vol.21, no. 1 , pp. 21-25.

[7] J. Jia, "The Practice of 'time and space effect' in soft clay excavation," Chinese Journal of Underground Space and Engineering, 2005, vol. 1, no. 4, pp. 490-493.

[8] Z. M. Wu and Y. M. Tu, "Space effect of soil-nailing excavation protection," Rock and Soil Mechanics, 2007, vol. 28, no. 10, pp. 2178-2182.

[9] G. P. Wang, G. B. Liu, "Finite element analysis of the deformation of deep excavations considering time-space effect in soft soils," China Civil Engineering Journal, 2009, vol. 42, no. 4, pp. 114-118.

[10] G. N. Zhou, "Time-space effect analysis and control of deep foundation excavation in soft soil," Chinese Journal of Underground Space and Engineering, 2014, vol. 19, no. S1, pp. 1653-1658.

[11] Y. J. Li, C. H. Wang, Q. Sun and Y. Xing, "Influence of subway deep foundation excavation on surrounding ground settlement," Journal of Liaoning Technical University (Natural Science), 2017, vol. 36, no. 4, pp. 387-390.

[12] E. M. Goldratt, Critical Chain, NY: North River Press, 1997. 
[13] Z. Y. Zhang and S. Wu, "Incidencedegree model of interval grey number based on whitening weight function," Chinese Journal of Management Science, 2015, vol. 23, no. 01, pp. 154-162.

[14] Y. W. Zhang, G. F. Ma and J. X. You, "Flexible control of tunnel construction schedule based on PDCA," Chinese Journal of Underground Space and Engineering, 2005, no. 5, pp. 733-736.

[15] Z. Zhang, G. X. Chen, and M. M. Jiang, "Application of progress control of subway station based on Critical Chain Project Management," Journal of Engineering Management, 2017, vol. 31, no. 5, pp. $112-116$

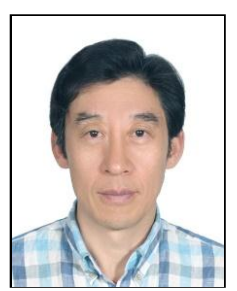

Guangming $\mathbf{Y u}$ received the bachelor degree in mine surveying from Liaoning Technical University, Fuxin, China in 1986, master degree in mine surveying from Liaoning Technical University, Fuxin, China in 1988, doctor degree in engineering mechanics from China University of Mining and Technology in 1997, and postdoctoral research at Silesica University of Technology, Poland in 1998, respectively.

He worked as an associate professor, a professor, a supervisor of master and doctor in Liaoning Technical University, Fuxin, China from 1997 to 2000. He has been a professor of School of Civil Engineering at Qingdao University of Technology, Qingdao, China from 2001, he is also a national candidate of the New Century Talents Project in China, the specialist who enjoys special subsidies from the State Council of the people's Republic of China and the director of Engineering Technique Research Center of Rock Mass Damage and Ground Subsidence Control and Treatment, Shandong Province. He has won 6 national, provincial and ministerial awards, such as the 2nd prize of national science and technology progress.

Prof. Yu presided over 65 items of the National Natural Science Foundation, Sino-Russian International Cooperation and metro construction projects. His primary research interests in underground engineering construction, building health maintenance and disaster monitoring of civil engineering.

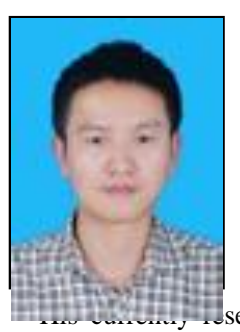

Xiankun Zeng is now a PhD student in the School of Civil Engineering of Qingdao University of Technology in China. In 2018, he received the master degree in civil engineering from the College of Architecture and Civil Engineering of Xinjiang University in China. In 2015, he received the bachelor degree in civil engineering from the College of Post and Telecommunication of Wuhan Institute of Technology in China.

earch interests in geotechnical engineering, including deformation monitoring of tailing dam and the influence analysis of metro tunnel or foundation pit construction on surrounding environment.

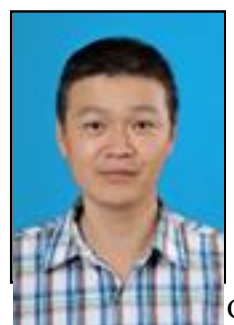

Yongjun Qin received the bachelor degree in industrial and civil construction engineering from Xinjiang University, Urumqi, China in 1994, master degree in industrial engineering from Xi an University of Architecture and Technology, Xi an, China in 2008 , respectively.

He worked as an associate professor, a professor, supervisor of master and assistant dean in Xinjiang University, Urumqi, China from 2009. He has been a Cola lege of Architecture and Civil Engineering of Xinjiang University, Urumqi, China from 2016; he is also a director of Xinjiang Society of Civil and Architecture.

Prof. Qin has mainly researched on the construction project management; he presided over and participated in more than 10 items of scientific research projects, including the urban rail transit project.

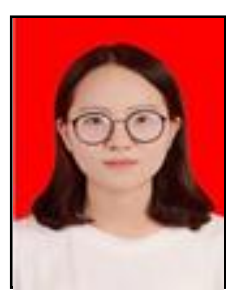

Yongning $\mathbf{L i}$ received the bachelor degree in engineering management from Xinjiang University, Urumqi, China in 2017. At present, she is a master degree candidate in the College of Architecture and Civil Engineering of Xinjiang University in China.

Her currently research interests in management research about geotechnical engineering, such as optimization of construction schedule in deep foundation pit.

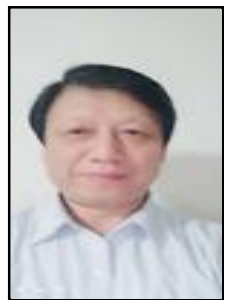

Yingnian Yu received the bachelor degree in construction engineering from the People's Liberation Army University of Science and Technology in 2001, he engaged in the ground building protection of three unders in coal mining in Jixi Mining Bureau from 1983 to 1993 . Since 1993 , he has worked as a construction technician and project manager in Zhongqi Construction Group Co., Ltd., engaged in project construction and project management.

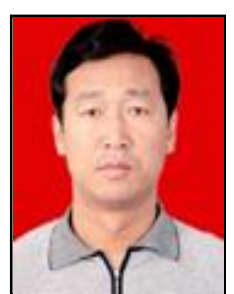

Xianguo Meng received the bachelor degree in engineering management from Lanzhou Jiaotong University, Lanzhou, China in 2014.

$\mathrm{He}$ engaged in the railway construction in China Railway Urumqi Group Co., Ltd. from 1992 to 2012 , working in Engineering and Technology Department and Safety Quality Department of Urumqi Urban Rail Group Co., Ltd. from 2012 to 2017. Since 2017, he has worked as a minister of safety and quality in Xinjiang Wujing Infrastructure Construction Management Co., Ltd. 